\title{
Information Pour Le Développement Durable De La Santé - Cas De L'usage Des Réseaux Sociaux Dans La Lutte Contre Le Cancer De La Prostate En Côte d'Ivoire
}

\author{
Gnamien Kouamé Pascal \\ Enseignant Chercheur à l'Institut des Sciences et Techniques de la \\ Communication, ISTC Polytechnique, Abidjan
}

Doi: 10.19044/esj.2018.v14n3p460 URL:http://dx.doi.org/10.19044/esj.2018.v14n3p460

\begin{abstract}
Prostate cancer is a pathology that causes casualties among the male population in Côte d'Ivoire. This pandemic kills silently but can be cured at the early stage of detection. All the actors in the fight against this pathology have mainly focused on the classical channels of communication and nonmedia. In the era of the information society, there is, however, a culture of digital technology in Côte d'Ivoire where the use of social networks is now part of the habits. While mass communication campaigns hinder efforts to raise awareness for early detection, more discreet and more customizable social networks have the advantage of reaching men who feel demeaned and humiliated when it comes to the «shameful, humiliating and dishonorable» disease of prostate cancer. The objectives of this research are to assess the impact of prostate cancer awareness campaigns on male population through traditional channels of communication and to analyze the determinants of the evolution of prostate cancer, despite all actions taken to propose solutions to curb the evolution of the disease. With the quantitative and qualitative approaches, the hypothesis that shows that there is a significant relationship between the evolution of prostate cancer among the male population and the late detection of this disease was confirmed. The communication strategies proposed by the various actors will then be effective, provided that social networks are associated with conventional media and non-media.
\end{abstract}

Keywords: Cancer, Prostate, Awareness, Social Networks, Male population

\section{Résumé}

Le cancer de la prostate est une pathologie qui fait des victimes au sein des sujets masculins en Côte d'Ivoire. Cette pandémie qui tue silencieusement peut être guérie grâce à un dépistage précoce. Tous les acteurs dans la lutte 
contre cette pathologie ont essentiellement mis l'accent sur les canaux classiques de communication et hors médias. À l'ère de la société de l'information, il s'établit cependant une culture du numérique en Côte d'Ivoire, où l'usage des réseaux sociaux fait désormais partie des habitudes. Si les campagnes de communication de masse freinent les efforts de sensibilisation en vue d'un dépistage précoce, les réseaux sociaux, plus discrets et plus personnalisables, ont l'avantage de toucher davantage les sujets masculins qui se sentent rabaissés et humiliés lorsqu'il est question de la maladie « honteuse, humiliante et déshonorante » du cancer de la prostate. Cette recherche a pour objectifs, d'une part, d'évaluer l'impact des campagnes de sensibilisation contre le cancer de la prostate sur les hommes à travers les canaux classiques de communication et d'autre part, d'analyser les déterminants del'évolution du cancer de la prostate malgré toutes les actions menées, en vue de proposer des solutions pour freiner l'évolution de la maladie. Avec les approches quantitative et qualitative, l'hypothèse qui stipule qu'il existe un lien entre l'évolution du cancer de la prostate et le dépistage tardif de cette maladie, est confirmée. Les stratégies de communication proposées par les différents acteurs pourront être efficaces à condition que les réseaux sociaux soient associés aux médias classiques et hors-médias.

Mots-clés : Cancer, Prostate, Sensibilisation, Réseaux Sociaux, Population Masculine

\section{Introduction}

Caractérisé par une prolifération incontrôlée de cellules qui envahissent et détruisent les tissus et organes environnants, le cancer de la prostate est une maladie cancéreuse (tumeur) qui touche la prostate, une glande exclusivement masculine jouant un rôle essentiel au niveau reproducteur.

À l'échelle mondiale, le cancer de la prostate est le deuxième cancer le plus répandu chez l'homme après celui du poumon ; il représente aujourd'hui $25 \%$ des cancers masculins. Selon les prévisions des experts en santé, 1/8 des hommes développeront un cancer de la prostate avant l'âge de 75 ans, et le nombre de nouveaux cas de cancer de la prostate augmente en moyenne de 4,8\% par an (allafrica.com, 2008).

Aucun pays n'est épargné, plusieurs personnes sont atteintes et le mal ne cesse de prendre de l'ampleur. Chaque année, plus de 40.000 hommes sont touchés, c'est la première cause de mortalité chez les hommes (allafrica.com, 2008). Face au fléau, Abecassis, Ameur et Debre (2001), Ravery (2010), Lenois (2011), Blum, Scholz et Joyeux (2012) Camilleri (2012), Benatta (2016) présentent le cancer et les modes de prévention de celui-ci, alors que Hay et Joyeux (2011), Schwartz (2016), Canavy (2014), Burnel et Gerson (2017) présentent le dépistage, le diagnostic et les options de traitement. Hay 
et Joyeux (2011), Camilleri (2012), Correa (2016), Greson (2017), Burnel et Gerson (2017) quant à eux, mettent en commun leur expérience pour apporter pas à pas, dans une démarche cohérente, les réponses liées aux questions du diagnostic et du traitement du cancer de la prostate.

Cette situation est encore plus critique en Afrique au sud du Sahara. En effet, il est établi que le taux du cancer de la prostate est plus élevé chez les hommes d'origine noire africaine, à cause d'un problème génétique. Les statistiques de l'Organisation Mondiale de la Santé (OMS) sont alarmantes : $12,4 \%$ des 804 millions d'hommes de la région africaine développeront une pathologie du cancer avant d'atteindre l'âge de 75 ans, et $90 \%$ des cas surviendront avant l'âge de 40 ans (allafrica.com, 2008).

En Côte d'Ivoire, les dernières statistiques publiées le 17 octobre 2012 donnent les chiffres suivants : 19,9\% pour le cancer du sein, $15,4 \%$ pour le cancer du foie, $10,4 \%$ pour le cancer du col de l'utérus, $9,3 \%$ pour le cancer de la prostate et 7,2\% pour les hémophilies malignes (Zagbayou, 2017), et de 16.000 à 20.000 nouveaux cas de cancer seraient diagnostiqués (Cissé, 2017). Les Ivoiriens sont de plus en plus conscients que cette maladie est une réalité dans leur pays. C'est face à ce fléau que SEM Alassane Ouattara a inauguré le lundi 18 décembre 2017 le Centre National de Radiothérapie, pour lutter contre le cancer. Dans son discours, il a invité les populations à ne plus avoir peur du cancer, car il y a de réelles possibilités de guérison en cas de dépistage rapide (Latt, 2017:2). C'est justement sur ce point que se trouve le problème au niveau des hommes en Côte d'Ivoire : le dépistage.

En effet, la majorité des hommes ivoiriens ne connaissent pas leur statut à cause de l'insuffisance d'informations sur cette maladie, en dépit de l'accent particulier mis par l'Organisation Mondiale de la Santé (OMS) dans ses campagnes de prévention et sensibilisation, sur la prévention, le dépistage, le diagnostic précoce et les soins palliatifs pour la lutte contre le cancer. " Malgré toutes les campagnes d'information et de sensibilisation réalisées par le programme [national de lutte contre le cancer] et les ONG, les populations ne sont pas encore bien imprégnées du fait que la consultation précoce peut sauver une vie. Mieux les gens seront informés sur la pathologie, plus tôt ils consulteront un médecin. Aujourd'hui, la médecine dispose de toute la panoplie de moyens pour guérir le cancer [...] lorsqu'il est diagnostiqué précocement 》(Sinzé, 2017:6). La pandémie n'est donc plus incurable, cependant, elle gagne de plus en plus de terrain.

Au cours des campagnes de sensibilisation proposées par les acteurs de la lutte contre le cancer (PNLC, AILC), l'OMS, le Ministère de la Santé, par certains chercheurs (Koffi, 2006 ; Kouassi, 2007 ; Komenan, 2012), l'accent est toujours mis sur les médias classiques et hors médias de communication, essentiellement des push médias (l'information va en direction du récepteur). Cependant, selon la mentalité des hommes (la principale cible) en Côte 
d'Ivoire, le thème de la maladie de la prostate, parce qu' elle touche à l'appareil génital de l'homme, est sensible, plus précisément « humiliant», " honteux » et « déshonorant » et par conséquent «tabou ». Les hommes semblent prendre donc des distances et rester fermés aux différentes campagnes de sensibilisation.

Entretemps, les habitudes informationnelles et communicationnelles ont aussi évolué progressivement en Côte d'Ivoire. En effet, la société ivoirienne connaît de profondes mutations à l'ère de la société de l'information, précisément du numérique. La culture numérique y est irréversible. Le pays comptait en 2015 près de 600 cybercafés et 7 millions d'utilisateurs réguliers d'Internet (Gnamien, 2017:131). 90\% de la génération Y ayant atteint 18 ans, disposent d'un smartphone et $75 \%$ d'un PC ; 70\% ont l'intention de s'acheter un smartphone et $43 \%$ un PC (Deloitte, 2016) ; 69,31\% des terminaux utilisés pour le commerce en ligne sont des ordinateurs, 25,68\% des mobiles et 5,01\% des tablettes (kaymu.ci, 2015). En Côte d'Ivoire, 22\% des cyberconsommateurs, c'est-à-dire des lecteurs potentiels, sont âgés de 18 à 24 ans, 34\% d'entre eux ont entre 25 et 34 ans, $27 \%$ ont entre 35 et 44 ans, $11 \%$ entre 45 et 54 ans et 4,5\% sont âgés de 55 à 64 ans (kaymu.ci, 2015).

Ces chiffres de l'e-commerce donnent une idée de la cible qu'on pourrait toucher en ligne. En outre, ces nouveaux canaux numériques de communication, essentiellement des pull medias (le récepteur choisit luimême l'information à consommer) ont l'avantage d'être plus personnels et plus discrets face à la « honteuse » maladie (pour l'homme africain) du cancer de la prostate.

Si l'évolution de cette pathologie est due à l'insuffisance d'informations des populations masculines sur la maladie, le sujet se rapportant au sexe étant un tabou en Afrique, le mal, lui, gagne du terrain et il faut penser à l'éradiquer.

Ce constat nous a amené à choisir comme objet d'étude « L'information pour le développement durable de la santé - cas de l'usage des réseaux sociaux dans la lutte contre le cancer de la prostate en Côte d'Ivoire ". Il s'agit d'abord de faire l'état des lieux sur la connaissance du cancer de la prostate au sein de la population ivoirienne, les mesures de prévention de base et autres mesures de prévention; ensuite, présenter le niveau de prise en charge de cette maladie et enfin, proposer aussi des stratégies de communication orientées vers le numérique, plus anonyme, plus personnalisable et plus discrète, en vue de sensibiliser la population masculine, qui semble fermée, aux autres canaux, sur les dangers du cancer de la prostate.

En d'autres termes, cette étude s'inscrit dans l'optique d'évaluer le niveau de connaissance des populations sur le cancer de la prostate et l'importance de les sensibiliser en développant un autre pan de la e-Santé, la 
sensibilisation à travers le numérique (la e-Sensibilsation), en plus des canaux traditionnels de communication.

\section{Objectifs et hypothèses de recherche \\ Objectifs de recherche Objectifs principaux}

Les principaux objectifs sont premièrement d'évaluer l'impact sur les hommes des campagnes de sensibilisation contre le cancer de la prostate, à travers les canaux classiques de communication; et deuxièmement, d'analyser les déterminants de l'évolution du cancer de la prostate malgré toutes les actions menées, en vue de proposer des solutions pour freiner l'évolution de la maladie.

\section{Objectifs spécifiques}

Quant aux objectifs spécifiques, il s'agit de :

- déterminer le degré de connaissance de la maladie chez les hommes ;

- $\quad$ identifier le facteur entravant une communication efficace dans la lutte contre le cancer de la prostate ;

- déterminer la perception qu'ont les hommes à l'égard de cette pathologie ;

- $\quad$ élaborer sur pied une stratégie de sensibilisation de communication intensive, afin que le cancer de la prostate n'évolue plus en Côte d'Ivoire.

\section{Hypothèses de recherche Hypothèse principale}

Comme hypothèse, nous retenons pour cette étude, qu'il existe un lien entre l'évolution du cancer de la prostate et le dépistage tardif de cette maladie.

\section{Hypothèses spécifiques \\ Hypothèse spécifique 1}

Plus le cancer de la prostate est pour les hommes une maladie humiliante, déshonorante et honteuse, plus les messages véhiculés pendant les campagnes de sensibilisation par les canaux classiques de communication (des push médias) pour un dépistage sont difficilement assimilés.

\section{Hypothèse spécifique 2}

La stratégie de communication efficace dans la lutte contre le cancer de la prostate passe par les campagnes de sensibilisation à travers les canaux plus personnels et plus discrets du numérique (les réseaux sociaux), les pull médias. 


\section{Méthodologie}

\section{Identification des variables}

Hypothèse spécifique 1

Variable Dépendante : Assimilation difficile

Variable Indépendante : Conception qu'ont les hommes sur la maladie

Hypothèse spécifique 2

Variable Dépendante : Efficacité des campagnes de communication

Variable Indépendante : Choix des canaux plus personnels et plus discrets du numérique, les pull médias

\section{Indicateurs de variables}

Hypothèse spécifique 1

Assimilation difficile : non considération des messages, refus d'accepter la pathologie

Conception qu'ont les hommes sur la maladie : maladie humiliante, déshonorante et honteuse

\section{Hypothèse spécifique 2}

Efficacité des campagnes de communication : Plus de 50 fois par an Choix des canaux plus personnels et plus discrets du numérique, les pull médias : Facebook, Twitter, Instagram, LinkedIn, Medium, Periscope, Snapchat, Tumblr, Viadeo, YouTube, Google+.

\section{Site et participants à l'enquête}

L'enquête a été réalisée dans les limites de la capitale économique de Côte d'Ivoire, qu'est Abidjan. Abidjan est l'une des plus grandes villes de l'Afrique de l'Ouest francophone et est très peuplée, avec une population estimée à plus de 5 millions d'habitants, soit près de $20 \%$ de la population totale de la Côte d'Ivoire.

La population cible est constituée de 150 hommes vivant dans la ville d'Abidjan (surtout les biens portants) ayant 40 ans et plus. Nous avons choisi cette tranche d'âge en raison du fait que le cancer de la prostate est diagnostiqué généralement chez les hommes de plus 50 ans. De ce fait, nous avons une meilleure possibilité de sensibiliser cette population. L'enquête s'est déroulée dans les communes de Treichville, Yopougon, Plateau, Cocody et Abobo ; 30 enquêtés par commune ont été choisies.

\section{Instruments de recueil des données}

La recherche documentaire, le questionnaire et l'entretien ont été utilisés dans le cadre de cette étude, comme instruments de recueil des données. 


\section{Recherche documentaire}

Pour mener à bien notre étude, nous avons utilisé l'étude documentaire, en vue de recueillir et analyser les données existantes relatives au cancer de la prostate au sein du service Cancérologie du Centre Hospitalier Universitaire (CHU) de Treichville, du service Urologie du Centre Hospitalier Universitaire (CHU) Cocody, de l'Association Ivoirienne de la Lutte contre le Cancer (AILC), des pull et push médias, ainsi que des rapports de professionnels de la santé sur le cancer de la prostate.

\section{Questionnaire}

Nous avons administré le questionnaire à notre population cible. Il nous a permis de quantifier les variables explicatives de l'évolution du cancer de la prostate malgré les actions menées, et de recueillir des suggestions sur les actions à mener en vue d'une meilleure sensibilisation des populations masculines. Une fois l'enquête achevée, nous avons procédé à la codification, au dépouillement et à la présentation des données recueillies. Ainsi, l'ordinateur, grâce à plusieurs logiciels (Sphinx, ord, Excel), a permis de traiter les données de l'enquête. D'une part, le tri à plat, qui consiste à recenser dans des colonnes les variables, a été utilisé pour une analyse simple des données. Le total et les pourcentages sont calculés par la règle de trois et d'autre part, le tri croisé, qui permet de croiser, de mettre en corrélation les réponses obtenues lors de l'administration du questionnaire, afin d'approfondir l'analyse, a également capté notre attention. En outre, nous expliciterons nos résultats par les différents tableaux et interprétations.

\section{Entretien}

Nous avons eu recours à l'entretien semi-directif. Les personnes interviewées ont directement répondu à des questions précises structurées. Cet exercice vise à obtenir des réponses spontanées et franches. L'entretien a permis de rechercher les explications psychologiques du comportement de l'homme ivoirien face à la maladie du cancer de la prostate, et les motifs de ses attitudes vis-à-vis des moyens de communication. Ceci dans le but de déceler ses habitudes ou ses motivations sur lesquelles il est possible plus tard, dans les suggestions, d'exercer une influence dans le choix d'une stratégie appropriée pour les campagnes de sensibilisation sur la maladie du cancer de la prostate.

\section{Résultats}

Caractéristiques sociodémographiques des enquêtés

Tableau 1 : Tranche d'âge

\begin{tabular}{|c|c|c|}
\hline Tranche d'âge & $\begin{array}{c}\text { Hommes } \\
\text { participants }\end{array}$ & Pourcentage \\
\hline
\end{tabular}




\begin{tabular}{|c|c|c|}
\hline 30 ans -40 ans & 56 & $37,3 \%$ \\
\hline 41 ans -50 ans & 14 & $9,3 \%$ \\
\hline 51 ans -60 ans & 48 & $32,0 \%$ \\
\hline 61 ans et plus & 32 & $21,3 \%$ \\
\hline Total & 150 & $100 \%$ \\
\hline
\end{tabular}

Source : Données d'enquête Juin 2016

Selon les données du tableau, 37\% des hommes interrogés ont un âge compris entre 30 et 40 ans, et 32\% dont l'âge est compris entre 51 et 60 ans.

Tableau 2 : Statut Socioprofessionnel

\begin{tabular}{|c|c|c|}
\hline Statut Socioprofessionnel & Participants & Pourcentage \\
\hline Fonctionnaire & 56 & $37,3 \%$ \\
\hline Profession libérale (à son propre compte) & 41 & $27,3 \%$ \\
\hline Salarié du privé & 48 & $32,0 \%$ \\
\hline Étudiant/Élève & 3 & $2,0 \%$ \\
\hline Sans emploi & 2 & $1,3 \%$ \\
\hline Total & 150 & $100 \%$ \\
\hline
\end{tabular}

Source : Données d'enquête Juin 2016

Les données du tableau 2 nous révèlent que parmi les enquêtés, $38 \%$ sont fonctionnaires, $32 \%$ sont salariés du privé et $27 \%$ ont des professions libérales.

\section{État des connaissances sur le cancer de la prostate}

Tableau 3 : Connaissance du cancer de la prostate

\begin{tabular}{|c|c|c|}
\hline Connaissance du & \multicolumn{2}{|c|}{ Participants } \\
\cline { 2 - 3 } $\begin{array}{c}\text { cancer de la } \\
\text { prostate }\end{array}$ & Nombre & Pourcentage (\%) \\
\hline Oui & 42 & $28,0 \%$ \\
\hline Non & 108 & $72 \%$ \\
\hline Total & 150 & $100 \%$ \\
\hline
\end{tabular}

Source : Données d'enquête Juin 2016

Les données du tableau 3 nous montrent que $72 \%$ des hommes interrogés ne connaissent pas le cancer de la prostate. Cela veut dire que la majorité des enquêtés ignorent ce qu'est cette maladie.

Tableau 4 : Vecteurs de communication sur le cancer de la prostate

\begin{tabular}{|c|c|c|}
\hline $\begin{array}{c}\text { Vecteurs de communication } \\
\text { sur le cancer de la prostate }\end{array}$ & Participants & Pourcentage \\
\hline Radio & 5 & $12 \%$ \\
\hline Télévision & 10 & $14 \%$ \\
\hline
\end{tabular}




\begin{tabular}{|c|c|c|}
\hline Affiche & 0 & $0,0 \%$ \\
\hline Presse & 0 & $0,0 \%$ \\
\hline Internet & 15 & $10,70 \%$ \\
\hline Bouche à oreille & 43 & $8,70 \%$ \\
\hline $\begin{array}{c}\text { Lors d'une campagne de } \\
\text { sensibilisation }\end{array}$ & 2 & $1,33 \%$ \\
\hline Total & 42 & $100 \%$ \\
\hline
\end{tabular}

Source : Données d'enquête Juin 2016

Les enquêtés ayant répondu qu'ils connaissent le cancer de la prostate, affirment l'avoir connu par l'intermédiaire de la télé (14\%), de la radio (12\%) et d'internet $(10,70 \%)$. Ceux qui affirment ne pas connaitre la maladie représentent $72 \%$ de l'échantillon. Concernant internet, il s'agit de connaître les sites qui sont visités.

Tableau 5 : Les sites web utilisés

\begin{tabular}{|c|c|c|}
\hline Sites visités & Participants & Pourcentage \\
\hline Abidjan.net & 17 & $11 \%$ \\
\hline educarrière.net & 30 & $20 \%$ \\
\hline Facebook & 68 & $45 \%$ \\
\hline Twitter & 35 & $24 \%$ \\
\hline Total & 150 & $100 \%$ \\
\hline
\end{tabular}

Source : Données d'enquête Juin 2016

Les données du tableau 5 démontre que les enquêtés (69\%) visitent davantage les réseaux sociaux (Facebook - $45 \%$ et Twitter - 24\%) que les autres sites internet.

Ces résultats corroborent les conclusions de l'étude empirique de Odi (2014) et de Médiamétrie (2017). En effet, sur un échantillon de 200 personnes dans les 10 communes d'Abidjan parmi lesquelles il y a 51,50\% d'hommes et $48,50 \%$ de femmes, on observe que $100 \%$ des interviewés utilisent internet et 98\% les réseaux sociaux (Odi, 2014). 49,50\% utilisent ces derniers comme plateforme d'échange, $46,50 \%$ comme plateforme de communication, 40,50\% comme lieu de rencontre, $9 \%$ l'utilisent pour faire autre chose et $7 \%$ pour des interactions sociales.

Les plus connus sont Facebook, à 100\%, suivi de Twitter à 65,50\%, LinkedIn à 40,50\%, hi5 à 26,50\% et Viadeo à 50\% (Odi2014:51). 96,50\% des enquêtés possèdent un compte Facebook, 25\% un compte Twitter, 15\% YouTube et 18,5\% LinkedIn (Odi, 2014:53). 31,5\% utilisent les réseaux 
sociaux depuis plus de 5 ans, $23 \%$ depuis plus de 4 ans et $18 \%$ depuis plus de trois ans. Quant à leur utilisation par semaine, 36,50\% en font usage 3 fois, $20,50 \% 4$ à 6 fois et $31 \%$ entre 7 et 10 fois. $25,50 \%$ des interviewés y passent plus de 5 heures, $27 \%$ plus de 3 heures et $25,50 \%$ plus de 5 heures. $63,50 \%$ y vont pour partager des informations et $78 \%$ pour discuter. Pour finir, $46 \%$ des enquêtés jugent les réseaux sociaux importants (Odi, 2014:60).

En outre, interrogés en 2016 sur leurs équipements multimédias et leurs usages d'Internet, près de 9 foyers sur $10(87,7 \%)$ possèdent un smartphone en Côte d'Ivoire, c'est l'équipement le plus répandu et en forte progression : près de $16 \%$ de plus en 6 mois. Il se situe loin devant l'ordinateur $(19,2 \%)$ et la tablette $(14,5 \%)$. Près d'une personne sur cinq $(19,4 \%)$ se connecte à Internet tous les jours. Les usages des réseaux sociaux sont en progression, avec $44,5 \%$ d'inscrits à au moins un réseau social, Facebook arrivant en tête, devant Google+, Twitter et Instagram (Médiamétrie, 2017).

Tous ces chiffres montrent qu'il s'établit indéniablement en Côte d'Ivoire une culture du numérique et plus précisément, de l'usage des réseaux sociaux.

\section{Cancer de la prostate, une maladie nécessitant une prise en charge}

Les résultats révèlent que le cancer de la prostate est le deuxième cancer le plus répandu chez l'homme, dans le monde. Ainsi, chaque année, plus de 40.000 hommes sont touchés par le cancer de la prostate, c'est la première cause de mortalité chez les hommes. Le nombre de nouveaux cas de cancer de la prostate augmente en moyenne de 4,8\% par an. En Côte d'Ivoire, le cancer de la prostate est le premier cancer de l'homme (15,8\%), avant celui du foie. La maladie touche 30 habitants sur 100.000 et est une tumeur maligne qui touche les hommes à partir de 40 ans et plus. Les moyens de lutte et de prévention sont nombreux, cependant, dans notre pays, le manque d'infrastructures ne permet pas une bonne prise en charge thérapeutique des malades. Soucieux du bien-être et de l'ampleur de cette pathologie, le Ministère de la Santé et de l'Hygiène Publique a mis en place le Programme National de Lutte contre le Cancer (PNLC), qui lutte pour la sensibilisation et la réduction des cas de cancer en Côte d'Ivoire. Certaines Organisations NonGouvernementales (ONG) et associations militent également dans ce sens, notamment l'Association Ivoirienne de Lutte contre le Cancer.

Procéder à un dépistage régulier du cancer de la prostate participe fortement au traitement efficace de ce type de tumeurs. Même s'il ne s'agit pas d'une prévention à proprement parler, le dépistage d'un cancer est toujours important. En effet, en détectant très rapidement un cancer, avant même l'apparition des premiers symptômes, le cancer prostatique peut être très rapidement pris en charge. Cela augmente considérablement les chances de bons résultats thérapeutiques et donc le pronostic. 
Deux tests peuvent être utilisés par le médecin pour tenter de dépister de manière précoce un cancer de la prostate qui n’ont aucun symptôme : le toucher rectal consiste à introduire un doigt, en général un index, protégé par un gant lubrifié par la vaseline, dans l'anus du patient ; le test de l'antigène prostatique spécifique (APS) permet, de son côté, de mesurer la quantité d'APS (protéine fabriquée par les cellules de la prostate) dans le sang. Le test sanguin de l'antigène prostatique spécifique est utilisé pour apprécier la valeur d'une enzyme habituellement produite exclusivement par les cellules de la prostate.

\section{État d'avancement du cancer de la prostate en Côte d'Ivoire}

Lorsque le cancer de la prostate est diagnostiqué, on définit un traitement et des rendez-vous pour suivre le malade et voir comment celui-ci répond au traitement. Il n'existe pas un dépistage de masse mais plutôt individuel, car le dépistage demande plusieurs étapes, en fonction de chaque individu.

\section{Niveau de prise en charge}

Il n'existe pas encore en Côte d'Ivoire des prises en charge sociale pour les malades atteints du cancer de la prostate. Tous les frais médicaux reviennent à la charge des malades. Pour le traitement de la maladie, il a été indiqué que c'est le service d'urologie qui s'occupe de la maladie en premier lieu, mais à un certain stade, lorsque la maladie devient générale, les services de médecine et de cancérologie peuvent s'en occuper, mais toujours sous supervision d'un urologue.

Tableau 6A : Information sur le service de cancérologie et sa prise en charge

\begin{tabular}{|c|c|c|}
\hline \multirow{2}{*}{$\begin{array}{c}\text { Connaissance du } \\
\text { centre de } \\
\text { cancérologie }\end{array}$} & Nombre & Pourcentage (\%) \\
\cline { 2 - 3 } & 42 & $28,0 \%$ \\
\hline Oui & 108 & $72 \%$ \\
\hline Non & 150 & $100 \%$ \\
\hline Total & Participants \\
\hline
\end{tabular}

Source : Données d'enquête Juin 2016

La lecture du tableau $6 \mathrm{~A}$ nous révèle que les enquêtés $(72 \%)$ ne connaissent pas le centre de cancérologie.

Tableau 6B : La prise en charge offerte au sein du Centre de Cancérologie

\begin{tabular}{|c|c|c|}
\hline Prise en charge centre & Participants & Pourcentage \\
\hline Non réponse & 107 & $72,0 \%$ \\
\hline Pas du tout satisfaisante & 32 & $23,30 \%$ \\
\hline Plutôt pas satisfaisante & 10 & $6,66 \%$ \\
\hline
\end{tabular}




\begin{tabular}{|c|c|c|}
\hline Plutôt satisfaisante & 0 & $0,00 \%$ \\
\hline Tout à fait satisfaisante & 0 & $0,00 \%$ \\
\hline Total & 42 & $100 \%$ \\
\hline
\end{tabular}

Source : Données d'enquête Juin 2016

Ce tableau montre que $23,3 \%$ des enquêtés trouvent la prise en charge du Centre de Cancérologie pas du tout satisfaisante.

\section{Actions de sensibilisation déjà menées sur la maladie}

Les actions déjà menées sont peu efficaces, car ce sont les non spécialistes qui tentent d'expliquer la maladie à la population, et l'on s'en rend compte lors des séminaires, que les malades sont souvent diagnostiqués à un stade avancé.

\section{Mesures de prévention de base du cancer de la prostate}

Comme tout cancer, il n'existe pas de causes clairement définies, on parle plutôt de facteurs favorisants, comme l'âge, la testostérone, les facteurs génétiques mais aussi l'environnement dans lequel vit l'individu.

Une bonne hygiène de vie, notamment une alimentation équilibrée et la pratique d'une activité physique, réduisent les risques de développer le cancer, y compris celui de la prostate. Il est donc nécessaire de suivre quelques règles simples pour rester en bonne santé : la vitamine $\mathrm{D}$ constitue un complément anticancéreux de la prostate. Il ne faut donc pas hésiter à favoriser la consommation de l'anguille, du saumon, de la sardine, de la sole, du thon, ou encore, de l'huile de foie de morue. Le thé vert serait un aliment anticancéreux intéressant, en raison de sa forte teneur en catéchines, des substances antioxydants. Celles-ci participeraient au blocage des tumeurs lorsqu'on consomme environ 5 ou 6 tasses de thé vert chaque jour.

Selon une étude américaine, la consommation trop importante de viande rouge et de charcuterie multiplie par deux les risques de développer un cancer de la prostate. Pas moins de 12 études concluent que le lait favorise l'apparition de cancers de la prostate. Selon ces travaux, les risques de développer un cancer de la prostate sont 1,5 à 5,5 fois plus importants chez les grands consommateurs de produit laitiers (allafrica.com, 2008).

\section{Discussion et Suggestions Discussion}

Le cancer de la prostate est le premier cancer chez l'homme, qui se contracte au-delà de 55 ans. Chaque année, plus de 40.000 hommes sont touchés par la maladie, et c'est la première cause de mortalité chez l'homme. Le nombre de nouveaux cas augmente en moyenne de $4,8 \%$ par an. 
Le cancer de la prostate est une tumeur maligne qui prend naissance dans les cellules de la prostate. Mais il peut arriver que certains hommes contractent la tumeur bien avant cet âge. La plupart des malades ignorent l'existence de ce cancer avant la manifestation de la maladie.

Il n'existe pas de causes clairement définies pour cette maladie, mais plutôt des facteurs favorisant ou non la maladie, parmi lesquels l'âge et l'environnement. Les moyens de prévention permettront de réduire les risques de développer la maladie. Il existe des mesures de prévention de base, en l'occurrence une bonne hygiène de vie et d'autres mesures de prévention pour éviter le cancer de la prostate. L'on constate dans les pays au sud du Sahara qu'il n'existe pas de prise en charge sociale pour les malades atteints du cancer de la prostate, ce qui rend leur suivi très difficile.

Notre étude nous a permis de confirmer les deux (2) hypothèses: la première, selon laquelle «Plus le cancer de la prostate est pour les hommes une maladie humiliante, déshonorante et honteuse, plus les messages véhiculés pendant les campagnes de sensibilisation par les canaux classiques de communication (des push médias) pour un dépistage, les atteignent difficilement » et la deuxième, qui stipule que «La stratégie de communication efficace dans la lutte contre le cancer de la prostate passe par les campagnes de sensibilisation à travers les canaux plus personnels et plus discrets du numérique (les réseaux sociaux), les pull médias ».

Par ailleurs, l'insuffisance de communication sur la maladie et l'ignorance du cancer de la prostate par les hommes, freinent le dépistage précoce de cette maladie.

La non-utilisation des réseaux sociaux (utilisés à $69 \%$ par la cible sur le net) par les acteurs dans les campagnes de sensibilisation dans la lutte contre la pathologie, réduit la portée de celles-ci. En effet, les sujets masculins considèrent le cancer de la prostate comme étant une maladie difficile à confesser et préfèrent se soigner dans la discrétion, en évitant les campagnes de sensibilisation de masse, non personnalisées et non discrètes. Par conséquent, il est important, voire nécessaire, d'associer les réseaux sociaux aux canaux classiques de communication dans la lutte contre ce cancer.

Les stratégies de communication proposées seront alors efficaces à condition que les réseaux sociaux soient associés aux médias et hors-médias.

La télévision a toujours été privilégiée par tous les acteurs en tant que média leader, un média de grand public par excellence, puissant et valorisant, à forte audience et avec une pénétration rapide dans les foyers. Une enquête réalisée en 2016 a montré que 88,3\% des habitants d'Abidjan et de Bouaké, soit 2,5 millions de personnes, consacrent par jour en moyenne 3h14 de leur temps à consommer la télévision (Médiamétrie, 2017). Elle est suivie par la radio, qui a une bonne pénétration des populations, une utilisation très pratique et ayant l'avantage d'être un média de proximité, avec la possibilité d'un 
feedback téléphonique. La même étude au niveau de la radio révèle en 2016, avec la même cible, que 54,3\% des habitants d'Abidjan et Bouaké (soit 1,55 millions) ont écouté la radio en moyenne $3 \mathrm{~h} 06$ par jour. La télévision et la radio restent donc de puissants vecteurs de communication (Médiamétrie, 2017).

L'affichage est aussi un média qui a une capacité assez forte pour véhiculer un message. Il viendra en appui aux deux précédents médias, car il a un grand public à fort impact ; il garantit une bonne répétitivité et permet une bonne sélectivité géographique. En outre, la presse quant à elle, présente une bonne sélectivité de la cible; elle nous permet d'augmenter le nombre de lecteurs.

Les hors media, à savoir les flyers, campagnes de mailing et sms, le porte à porte, les sites web, les gadgets (Tee-shirts, calendriers, porte-clés, stylos), les caravanes et les conférences ont été privilégiés par tous les partenaires dans la lutte contre le cancer (PNLC, AILC), l'OMS, le Ministère de la Santé, dans les campagnes de sensibilisation explicative, afin d'inciter les hommes au dépistage.

\section{Suggestions}

En plus des canaux traditionnels de communication et hors médias, il est fortement recommandé de les associer aux différentes campagnes de sensibilisation de la population masculine dans la lutte contre le cancer de la prostate. Les réseaux sociaux, comparés aux autres vecteurs de communication, ont l'avantage d'être plus discrets et plus personnalisables. Les sentiments de honte, d'humiliation et de déshonneur des sujets africains mâles face à la maladie, disparaîtront.

En outre, le corps médical doit s'impliquer davantage dans les campagnes de sensibilisation. Les actions que doit mener le gouvernement : en premier lieu, c'est de considérer le cancer de la prostate comme certaines maladies chroniques, et rendre sa prise en charge gratuite. Il faudra ensuite mettre en place des structures adéquates pour le traitement des malades et enfin, médiatiser la maladie afin d'amener les hommes à se faire dépister.

L'ensemble de ces suggestions sont résumées dans le tableau suivant :

Tableau 7 : Actions à mener pour inciter les hommes à faire le test de dépistage du cancer de la prostate

\begin{tabular}{|c|c|c|}
\hline Actions à mener & Participants & Pourcentage \\
\hline Sensibiliser davantage sur l'importance du dépistage & 36 & $24,0 \%$ \\
\hline Informer sur les causes et les dangers & 12 & $08,0 \%$ \\
\hline Éduquer les hommes à adopter une alimentation saine & 28 & $18,66 \%$ \\
\hline Créer d'autres centres spécialisés de cancérologie & 24 & $16,0 \%$ \\
\hline
\end{tabular}




\begin{tabular}{|c|c|c|}
\hline Améliorer la communication sur le cancer de la prostate & 30 & $20,0 \%$ \\
\hline Réduire le prix du traitement du cancer de la prostate & 20 & $13,33 \%$ \\
\hline Total & 150 & $100 \%$ \\
\hline
\end{tabular}

Source : Données d'enquête Juin 2016

Les enquêtés, à la lecture de ce tableau, suggèrent de sensibiliser davantage sur l'importance du dépistage (24\%), afin d'amener les hommes à se faire dépister et lutter efficacement contre cette maladie. Ceux qui optent pour l'amélioration de la communication sur le cancer de la prostate représentent $20,0 \%$, suivis de ceux qui préconisent l'éducation des hommes à une alimentation saine $(18,66 \%)$ et enfin, de ceux qui plaident pour la réduction du prix de traitement de cette maladie $(13,33 \%)$.

\section{Conclusion}

Le cancer de la prostate est une réalité dans la société ivoirienne. Son évolution est due au fait que les hommes ne font pas de dépistage précoce. C'est une maladie qui tue silencieusement, elle peut cependant être guérie lorsqu'elle est rapidement diagnostiquée. Le sujet se rapportant à cette maladie est cependant un «tabou », surtout en Afrique, à cause des pressions culturelles, sociologiques et morales.

En Côte d'Ivoire, le cancer de la prostate est le plus répandu cancer chez l'homme, avant celui du foie (allafrica.com, 2008). Si l'étude a permis de montrer l'existence d'associations qui luttent contre cette pathologie et s'occupent du bien-être de malades, la maladie reste cependant méconnue pour la plupart des hommes. Pour freiner son évolution, nous suggérons de sensibiliser sur les dangers que représente cette pathologie, afin de les amener à faire le dépistage précoce.

En effet, lorsqu'elle se déclenche, le coût de la molécule essentielle pour le traitement de la prostate est coûteux et inaccessible pour les malades. La molécule Avastin, nécessaire au traitement du cancer du sein, du col de l'utérus, de l'ovaire, de certains cancers du poumon, de cancers du côlon et du rectum et de la prostate, est estimé à 1.541.405 FCFA par séance, à raison d'une séance tous les 21 jours et ce, pendant un an. Le traitement peut s'étendre sur huit (8) ans (Sinzé, 2017). Faire connaître les coûts liés aux soins à la population masculine est un argument très important et efficace lors des différentes campagnes de sensibilisation. Ceci amènerait la population masculine à se faire dépister.

« La bataille contre ce cancer, c'est d'abord la bataille de l'information juste, de l'information qui n'effraie pas, qui donne confiance [...] » (Sinzé, 2017:6). Pour atteindre les objectifs de sensibilisation, nous proposons non seulement des actions à travers les médias (Télé, Radio, Affichages, Presse) et hors 
médias (caravane, conférence, ONG, etc.), la sensibilisation de proximité, mais aussi et surtout les canaux plus discrets que sont les réseaux sociaux (Facebook, Twitter...).

\section{References:}

1. Abecassis, J. P., Ameur, A. \& Debre, B. (2001). Tout savoir sur la prostate. Paris: Favre.

2. Bausard, T. (2014). Le guide de l'alimentation anti cancer: Comment certains aliments préviennent ou luttent contre le cancer. Paris.

3. Benatta, M. (2016). Le Cancer de la Prostate de A à Z. Paris.

4. Bickmann, J. (2017). La Prostate Et si vous appreniez à dompter votre prostate? Ce que tout homme quel que soit son âge doit savoir sur le maintien de la santé de sa prostate. Paris.

5. Blum, R., Scholz, M. \& Joyeux, H. (2012). Touche pas à ma prostate : Un guide essentiel pour faire face au cancer de la prostate. Paris.

6. Burnel, G. \& Gerson, C. (2017). Guérir le cancer de la prostate et des testicules avec la méthode Gerson. Paris.

7. Camilleri, J.-P. (2012).Le Cancer de la prostate: 100 questionsréponses. Paris.

8. Canavy, B. (2014). Votre prostate, comment la rajeunir et la guérir. Paris.

9. Clapp, L. (2014).Guérir la prostate - Sans médicaments ni opération. Paris.

10. Correa, J. C. (2016). 33 Recettes de Repas qui vous aideront à lutter contre le Cancer de la Prostate, augmenter votre énergie, et vous sentir mieux: La solution simple à vos problèmes de cancer. Paris.

11. Greson, L. (2017). 21 Jours Pour Guérir Votre Prostate: Comment Guérir Presque N'Importe Quel Problème De Prostate Sans Médicaments, Sans Chirurgie, Sans Examens Internes. Paris.

12. Hay, M.H. \& Joyeux, H. (2011). Le cancer de la prostate: Enrayer l'épidémie et les récidives. Paris.

13. Kammerer, U., Knoll,G. \& Schlatterer, C. (2014). Le Régime cétogène contre le cancer. Paris.

14. Lenois, M. (2011). Mieux connaitre le cancer de la prostate. Paris: Springer Verlag.

15. Ravery, V. (2010). Cancer de la prostate. Paris: Springer Verlag.

16. Schwartz, L.(2016).Cancer: un traitement simple et non toxique. Paris.

17. Deloitte (2016). Etude sur la maturité numérique des entreprises en Côte d'Ivoire. Rapport Final. Abidjan: Deloitte.

18. Gnamien, K. P. (2017). Le vote électronique en Côte d'Ivoire - un nouveau défi pour l'e-gouvernance. In: Revue Ivoirienne de Gouvernance et d'Etudes Stratégiques (RIGES). (1) 212-151. 
19. Komenan, A.A. (2012).Stratégie de communication pour la lutte contre le cancer du col de l'utérus en Côte d'Ivoire. Mémoire de DESCOM, ISTC 2001-2012. Abidjan: ISTC

20. Kouassi, N. L. (2007). Quelle stratégie de communication pour l'usage du préservatif féminin en Côte d'Ivoire?Mémoire de DESCOM, ISTC2006-2007.Abidjan: ISTC

21. Koffi, Y. M. J. (2006). Quelles actions de communication en faveur du dépistage volontaire du VIH/SIDA dans les cités universitaires d'Abidjan? Mémoire de DESCOM, ISTC2005-2006.Abidjan: ISTC

22. Médiamétrie (2017). L'audience de la TV, de la Radio et les usages Internet en Côte d'Ivoire au $2^{\text {nd }}$ semestre 2016. Paris.

23. Odi, A. M. A.-D. (2014). Usage des réseaux sociaux chez les jeunes ivoiriens, effet de mode ou éducation au média: cas de Facebook. Mémoire de Master 2, ISTC 2014-2015. Abidjan: ISTC.

24. Cissé, A. (2017). Lutte contre le Cancer / CHU de Cocody. Le président Ouattara inaugure le centre de radiothérapie aujourd'hui. In: le Patriote du 18/12/2017/2017, (5402) 8.

25. Latt, T. (2017). Après l'inauguration du centre National de Radiothérapie. Ouattara va faire très fort en 2018. In: le Patriote du 19/12/2017, (5403) 2.

26. Sinzé, T. (2017). Traitement du cancer: l'espoir est permis. In: Fraternité Matin du 29/12/2017, (15913) 8.

27. Sinzé, T. (2017). Interview. Pr Innocent Adoubi. Directeur du Programme national de lutte contre le cancer. In: Fraternité Matin du 4/01/2017, (15917) 6.

28. Zagbayou, F. A. (2017). Lutte contre le cancer. Un vieux rêve enfin réalisé. In: Fraternité Matin du 18/12/2017, (15904) 6.

29. Aliam.org (2017).Le cancer de la prostate, en ligne, disponible sur:www.aliam.org/cote-d-ivoire/ailc, [06.03.2017 à 15h32].

30. Allafrica.com (2017). Le cancer de la prostate, en ligne, disponible sur www.allafrica.com/cancer/prostate[06.04.2017 à 10h10].

31. Kaymu.ci (2015). Les terminaux, ordinateurs, mobiles et tablettes. https://www.kaymu.ci. Consulté le 03.02.2015 à 14h20.

32. Sante.gouv.ci (2017). Statistique du cancer de la prostate en Côte d'Ivoire, en ligne, disponible sur:www.Sante.gouv.ci/index. PHP ?page $=$ actu $\& I D=146$, [06.03.2017 à 16h42]. 